\title{
Reduced facet temperature in semiconductor lasers using electrically pumped windows
}

\author{
Abdullah Demir ${ }^{1 *}$, Seval Arslan², Sinan Gündoğdu², Atilla Aydınlı ${ }^{3}$ \\ ${ }^{1}$ Bilkent University, UNAM - Institute of Materials Science and Nanotechnology, Ankara 06800, \\ Turkey \\ ${ }^{2}$ Bilkent University, Department of Physics, Ankara 06800, Turkey \\ ${ }^{3}$ Uludağ University, Department of Electrical and Electronics Engineering, Bursa 16059, Turkey
}

\begin{abstract}
The self-heating of semiconductor lasers contributes directly to facet heating and consequently to the critical temperature for catastrophic optical mirror damage (COMD) but the existing facet engineering methods do not address this issue. Targeting this problem, we report experimental and modeling results that demonstrate a new method achieving facet temperatures significantly lower than the laser cavity temperature in GaAs-based high-power semiconductor lasers by using electrically isolated and pumped windows. Owing to monolithic integration, the method does not introduce any penalty on the efficiency and output power of the laser. Thermal modeling results show that the laser output facet can be almost totally isolated from heat generated in the laser cavity and near cold-cavity facet temperatures are possible. The method can be applied to single emitters, laser bars, and monolithically integrated lasers in photonic integrated circuits to improve their reliability and operating performance.
\end{abstract}

Keywords: semiconductor laser, high power laser diode, catastrophic optical mirror damage (COMD), facet temperature, facet cooling

\section{INTRODUCTION}

Semiconductor lasers with reliable high output power are of great interest to many applications, especially recently for direct-diode and fiber lasers with $\mathrm{kW}$-level output used in material processing [1]. The reliability and performance of these high-power systems are backed up by the reliability of the single emitters. Although laser powers exceeding $30 \mathrm{~W}$ from a $100 \mu \mathrm{m}$ wide broad area laser have been demonstrated $[2,3]$, the operating output power of these devices are much lower (i.e. below $20 \mathrm{~W}$ ) due to reliability limitation. Catastrophic optical mirror damage (COMD) of the output facet is a key failure mode that constrains the device reliability and scaling of the operating laser power [4]. COMD is a heating induced device failure mechanism and even higher operating laser powers may be possible if it can be avoided.

Despite a variety of methods used for facet passivation and engineering [5], and high electro-optical power conversion efficiencies of these devices, the temperature rise at the facets limits the reliability and operating power of high power edge emitting semiconductor lasers. Facet heating leads to the bandgap shrinkage and further facet absorption resulting in COMD. Many successful methods were reported in the literature to prevent facet mirror reaching the critical temperature of $\mathrm{T}_{\mathrm{c}}=120-160{ }^{\circ} \mathrm{C}$ that triggers COMD on the output facet [4]. These methods limit the temperature increase of the output facet and, consequently, its optical strength against COMD is improved. Non-absorbing mirrors (NAMs) and unpumped windows near output facets have been employed to reduce optical absorption and facet heating [6,7]. NAMs produced by quantum well-intermixing is a complex process with tight fabrication tolerances. In the unpumped window method, facet temperature was shown to be lower using $\sim 30 \mu \mathrm{m}$ long current blocking region due to reduced current density near facet [8]. Alternatively, ultra-high vacuum cleaving and passivation of the facets eliminates the surface states for higher COMD thresholds at the cost of higher complexity and expense of fabrication [9]. These approaches have accomplished effective results increasing $T_{c}$. Although eliminating the surface recombination and optical absorption are important to limit the facet heating as targeted in the previous studies, the self-heating of the laser cavity contributes directly to facet heating and $\mathrm{T}_{\mathrm{c}}$, and it is probably the dominant source of facet heating. However, the existing methods have not addressed this issue before. Targeting this problem, we report experimental and modeling results that demonstrate a new method $[10,11]$ achieving facet temperatures significantly lower than the laser cavity temperature in $9 \mathrm{xx}-\mathrm{nm}$ high power semiconductor

*abdullah.demir@unam.bilkent.edu.tr

High-Power Diode Laser Technology XVII, edited by Mark S. Zediker, Proc. of SPIE Vol. 10900, 109000R · @ 2019 SPIE · CCC code: 0277-786X/19/\$18 doi: $10.1117 / 12.2509896$ 
lasers by using electrically isolated and biased transparent windows. Integrating long transparent window regions, where the transparency is controlled by applied bias with very low injection current, the self-heat load of the laser cavity can be thermally isolated from the output facet. Facet temperature was reduced by $40 \%$ compared to that of the control sample. Reduced facet temperatures are even below the laser cavity temperature demonstrating facet cooling. The thermal modeling of the devices shows that the facet temperature of a semiconductor laser can be reduced significantly and near cold-cavity facet temperatures are possible. Owing to monolithic integration, the method does not introduce any penalty on the power conversion efficiency and output power of the lasers.

\section{EXPERIMENTAL DETAILS}

The standard and new two-section laser approach are schematically compared in Fig. 1. A two-section chip is divided into two regions by separating the top electrode into laser and window sections. The laser cavity can operate at high current levels and therefore generates very high heat. The bulk temperature of the high-power laser diodes increases due to the dissipated power, and hence, for a standard laser, the facet temperature can be even higher than the laser cavity. In contrast, in a two-section laser, the window section next to the facet, is biased to transparency and, so, operates at very low current levels with negligible heat generation. Such a device configuration allows keeping the high heat load of the laser cavity away from the heat sensitive output facet. By extending the cold laser output window, the heat generated in the laser section can be separated from the output facet.

a) Standard single section laser

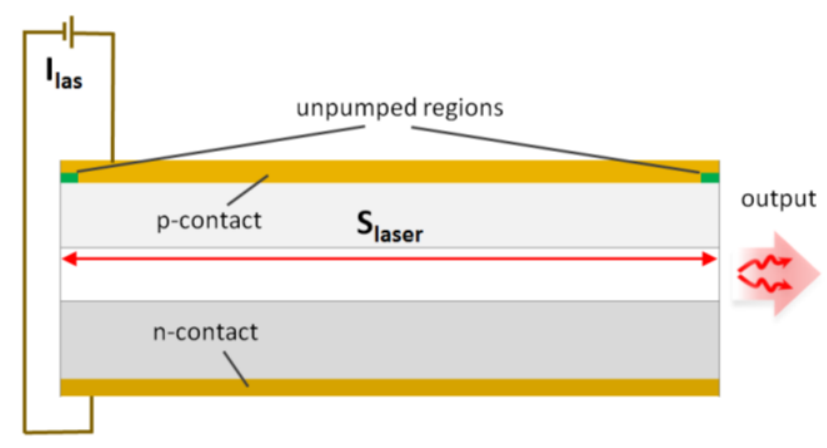

b) Two-section laser

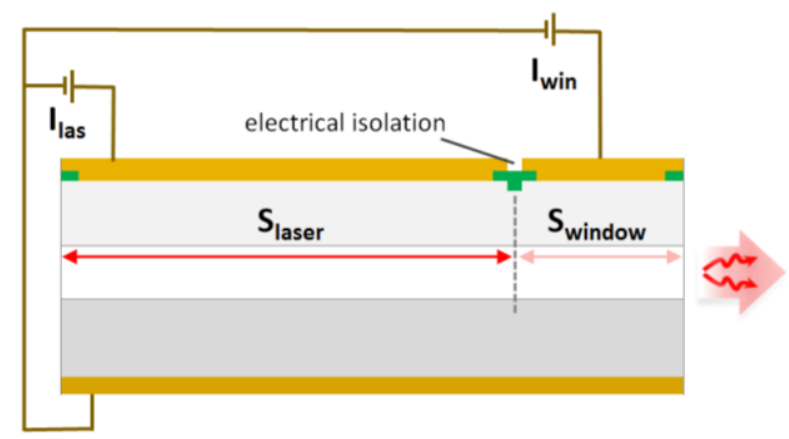

Figure 1. The schematic illustration of the side view of (a) standard single section laser, (b) developed two-section laser.

The epitaxial structure of our GaAs-based laser emitting at $915 \mathrm{~nm}$ is composed of $3000 \mathrm{~nm} \mathrm{n}$-AlGaAs cladding, $500 \mathrm{~nm}$ $\mathrm{n}$-AlGaAs waveguide, single InGaAs quantum well layer, $500 \mathrm{~nm}$ p-AlGaAs waveguide, $1000 \mathrm{~nm}$ p-AlGaAs cladding and $100 \mathrm{~nm}$ GaAs contact layer [12]. In this work, $5 \mathrm{~mm}$ long and $100 \mu \mathrm{m}$ wide broad area high power laser diodes with standard single-section and proposed two-section lasers were used to compare their performance and facet temperatures. Both lasers have $50 \mu \mathrm{m}$ unpumped current blocking windows near facets to prevent facet absorption due to excess carriers. No facet passivation and mirror coatings were applied. $200 \mathrm{~nm}$ thick $\mathrm{Si}_{3} \mathrm{~N}_{4}$ layer was deposited for passivation and unpumped windows. The two-section laser have trenches for electrical isolation between the laser and window sections so that they can be biased independently. We limited the depth of the isolation trench to $300 \mathrm{~nm}$ to preserve the mode profile and not to introduce optical losses. The electrical resistance between the lasing and window sections of two-section laser was measured as $69 \Omega$, which is $>1000$ higher than the series resistance of the laser. Hence, the carrier leakage between the laser and window sections is negligible. The lasers were cleaved and mounted epi-up on Cu-blocks.

Laser performance was evaluated under $\mathrm{CW}$ operation at room temperature for uncoated and epi-up lasers to simplify the observation of the facet cooling. The electro-optical power conversion efficiency of the lasers is around 52\% that is limited by uncoated facets and epi-up operation. As shown in Fig. 2, The dependence of the single-facet output power on the laser current is compared for the standard $(5 \mathrm{~mm}) \times(100 \mu \mathrm{m})$ and $(4 \mathrm{~mm}+1 \mathrm{~mm}) \times(100 \mu \mathrm{m})$ two-section lasers, where $1 \mathrm{~mm}$ is the length of the window section, at various window bias current levels. The power loss is around $15 \%$ when no bias is applied to the window section. The transparency and threshold current $\left(\mathrm{I}_{\mathrm{th}}\right)$ of $1 \mathrm{~mm}$ long cavity of our laser structure is estimated to be $76 \pm 7 \mathrm{~mA}$ and $257 \pm 28 \mathrm{~mA}$, respectively, using basic laser rate equations [13]. By applying an abovetransparency injection current of $100 \mathrm{~mA}$, the output power is recovered confirming that the absorption loss of the window section is eliminated, and transparency is established. The threshold current of the $5 \mathrm{~mm}$ long laser is $568 \mathrm{~mA}$ whereas the 
threshold current of $4+1 \mathrm{~mm}$ two-section laser with $\mathrm{I}_{\mathrm{win}}=100 \mathrm{~mA}$ is $463 \mathrm{~mA}$. This shows that the sum of the $\mathrm{I}_{\text {th }}$ and $\mathrm{I}_{\mathrm{win}}$ of the two-section laser is approximately equivalent to the $\mathrm{I}_{\text {th }}$ of the standard laser for the same total device length. The threshold current of the two-section laser decreases with an increase of the window section bias current since the spontaneous emission in the window section contributes to the gain in the laser section. Hence, the sum of $I_{\text {th }}$ and $I_{w i n}$ gives the actual threshold current of the two-section lasers.

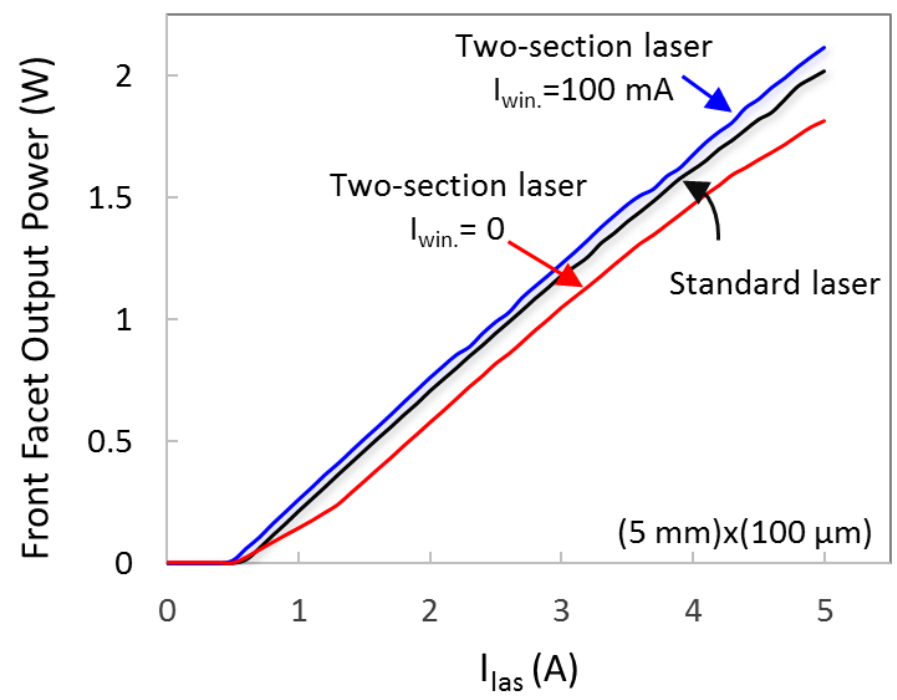

Figure 2. The front facet laser output power vs. laser current for the standard and two-section laser $\left(\mathrm{I}_{\mathrm{win}}=0\right.$ and $\left.100 \mathrm{~mA}\right)$.

Facet temperatures of the standard and two-section laser diodes were compared for emitters on the same bar. CMOS-based thermoreflectance method [14, 15] shown in Fig. 3(a) was used to obtain the temperature map of the facets. An LED at $450 \mathrm{~nm}$ was used as a probe light and directed onto the laser facet through a microscope objective (20x, 0.40 NA). Lasers were tested at room temperature up to 5 A laser injection current $\left(\mathrm{I}_{\mathrm{las}}\right)$. A current source diode driver (LDX-3600) was used under QCW current injection with $20 \mathrm{~ms}$ pulse duration and $20 \%$ duty cycle. Two-section lasers were tested under various

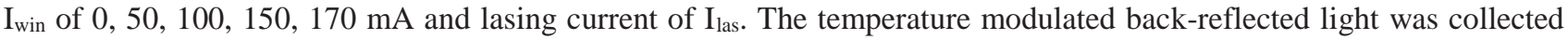
with a CMOS camera triggered by a diode driver and analyzed by a computer. The relative change in reflectivity was correlated with the temperature change given as

$$
\frac{\Delta R}{R}=\left(\frac{1}{R} \frac{\partial R}{\partial R}\right) \Delta T=\kappa \Delta T
$$

where $\kappa$ is the thermoreflectance coefficient and it depends on the laser material and probe light wavelength [14], $\mathrm{R}$ is the reflectivity of the sample material and $\mathrm{T}$ is the temperature. For the GaAs substrate of our laser structure, $\kappa$ was measured as $(3.2 \pm 0.6) \times 10^{-4}$ at $450 \mathrm{~nm}$ by modulating the laser temperature using a temperature controller. For our purpose of comparing the facet temperatures of standard and two-section lasers, possible differences between $\kappa$ of GaAs and epitaxial layers of the laser structure are not critical. Our measurements had $0.1^{\circ} \mathrm{C}$ temperature and $0.7 \mu \mathrm{m}$ spatial resolution. The spatial resolution allows us the characterize the temperature increase around the active region of the waveguide. Fig. 3(b) shows the facet temperature measurement result as a map for a $5 \mathrm{~mm}$ long standard laser at $\mathrm{I}_{\mathrm{las}}=3 \mathrm{~A}$. The active region has the highest temperature and heat spreads out both vertically and laterally. It is also clear that the right-hand side of the structure is cooler than that of the left due to its larger spacing from the edge of the chip compared to that of the left.

Figure 4(a) shows the temperature rise of the facet in the active region for the standard and two-sections lasers and compares them to the bulk of the laser cavity at $5 \mathrm{~A}$. Facet temperature for the standard laser increases by $59^{\circ} \mathrm{C}$, which is higher than its bulk temperature increase of $50^{\circ} \mathrm{C}$, as expected due to other contributions to the facet temperature such as non-radiative surface recombination and edge effects. For the two-section laser, the facet temperature increase stays below $37^{\circ} \mathrm{C}$ and reaches as low as $34.7^{\circ} \mathrm{C}$ at $170 \mathrm{~mA}$ of window current. Starting at $\mathrm{I}_{\text {win }}=100 \mathrm{~mA}$, which is above the estimated transparency current of $79 \mathrm{~mA}$, the facet temperature decreases slightly since optical absorption loss induced heating in the window is expected to be eliminated. Even though integrating a cold region between the laser and facet provides 
significant temperature reduction for the facet, it introduces an output power penalty if not biased above its transparency as shown in Fig. 4(b). The temperature is reduced by $40 \%$ at the facet and $30 \%$ compared to the bulk using the $1 \mathrm{~mm}$ long transparent window at $5 \mathrm{~A}$ laser injection current with $\mathrm{I}_{\mathrm{win}}=170 \mathrm{~mA}$.

(a)

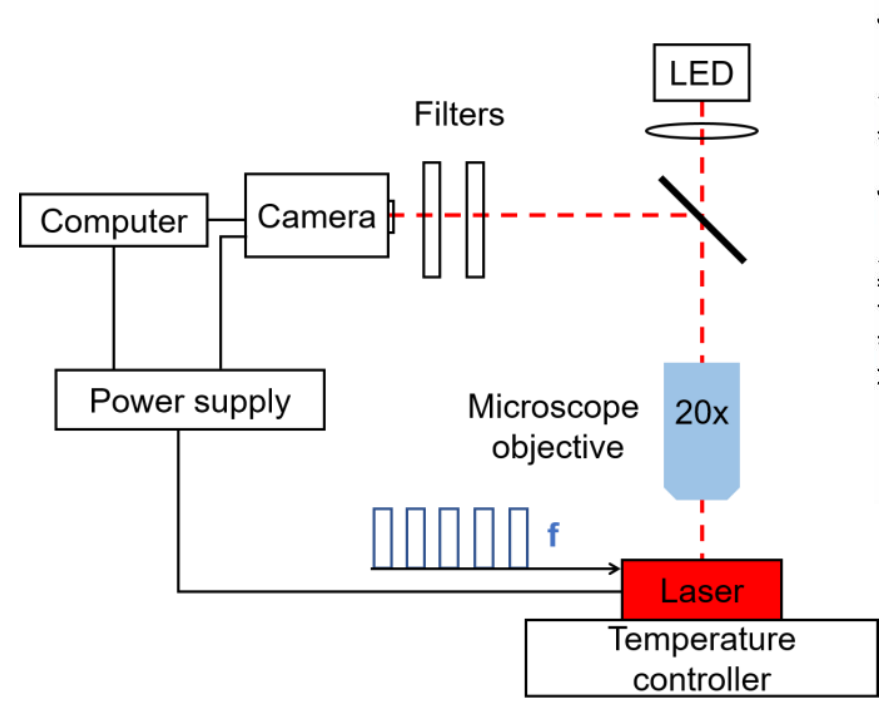

(b)

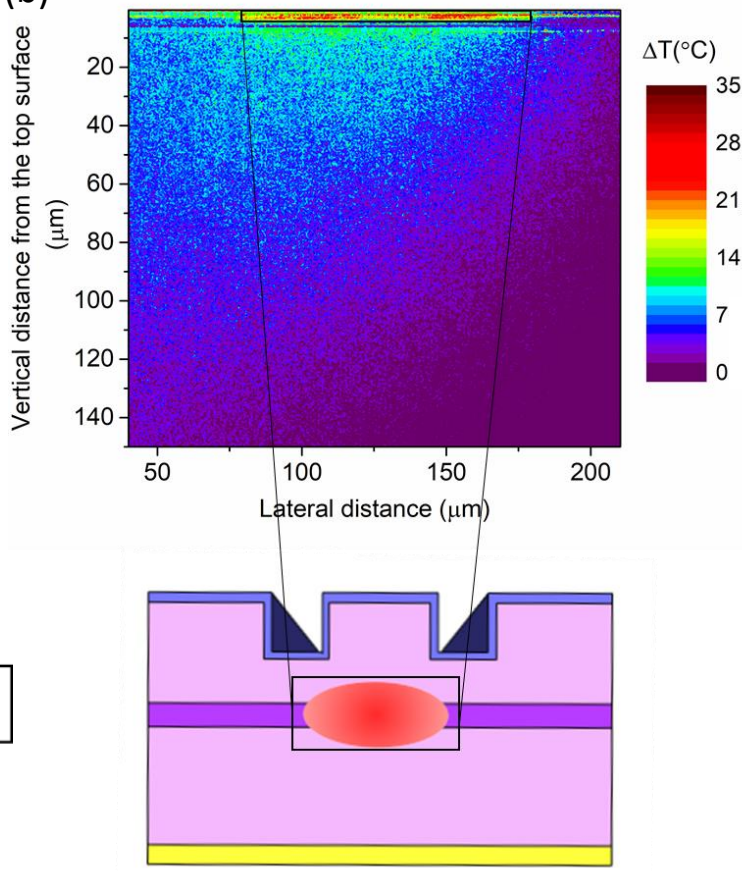

Figure 3. (a) Thermoreflectance setup used to measure the facet temperature of the lasers, and (b) the temperature map of a $5 \mathrm{~mm}$ long standard laser at $\mathrm{I}_{\mathrm{las}}=3 \mathrm{~A}$.

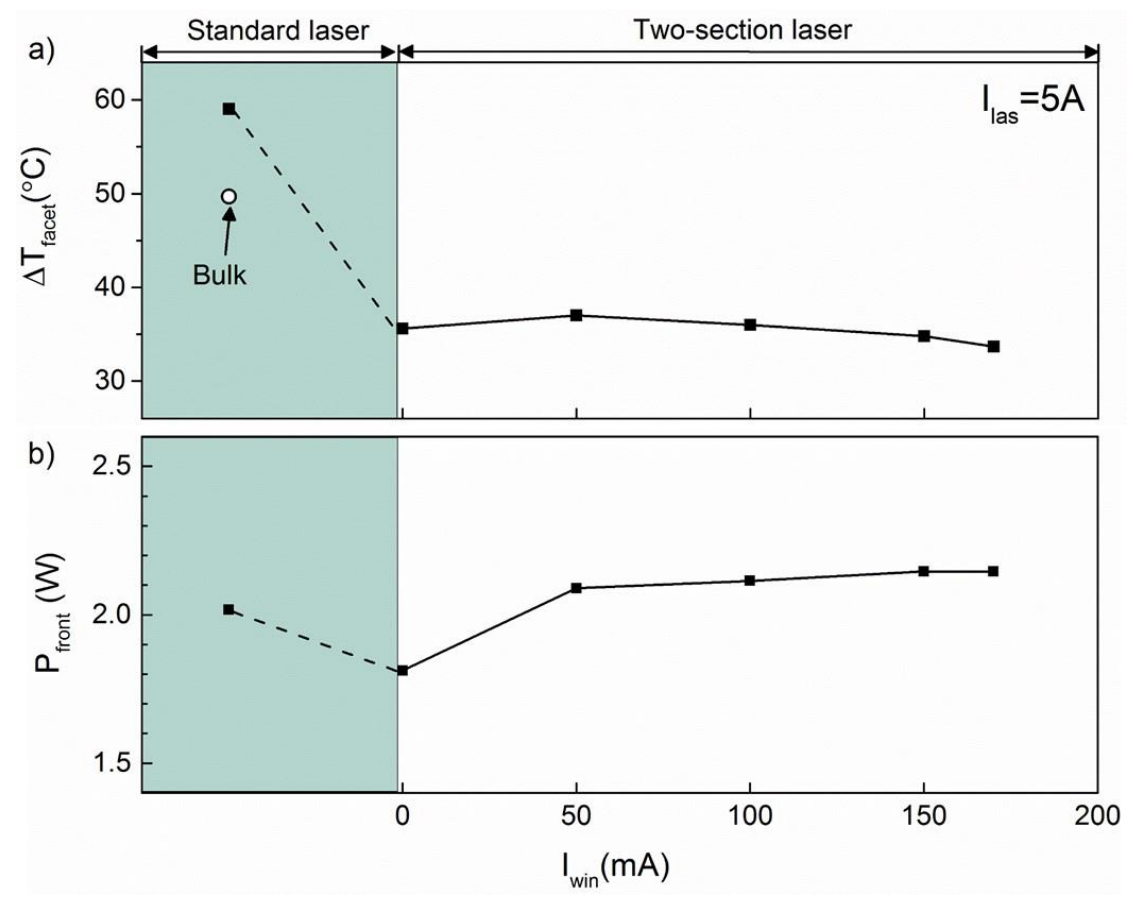

Figure 4. Comparing the active region facet temperature increase and front facet output power of standard, $(5 \mathrm{~mm}) \mathrm{x}(100 \mu \mathrm{m})$, and two-section, $(4 \mathrm{~mm}+1 \mathrm{~mm}) \mathrm{x}(100 \mu \mathrm{m})$, laser devices. 
(a)

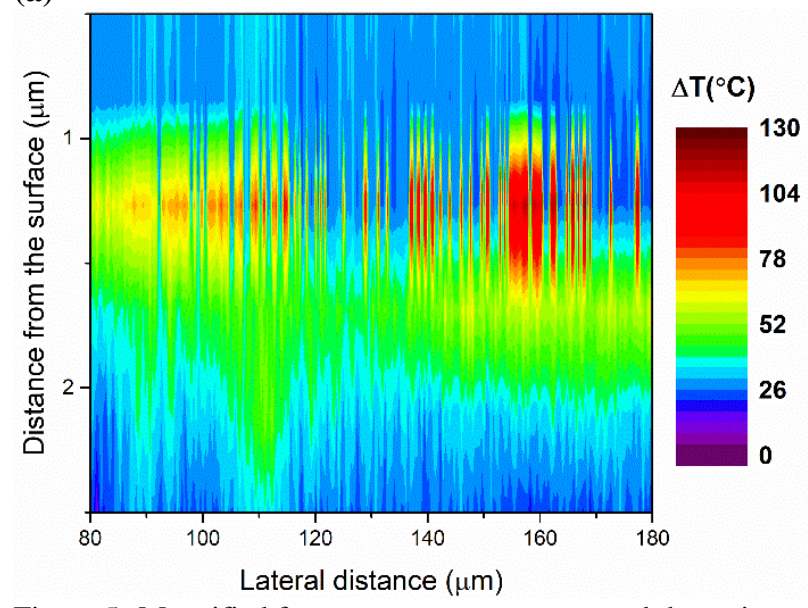

(b)

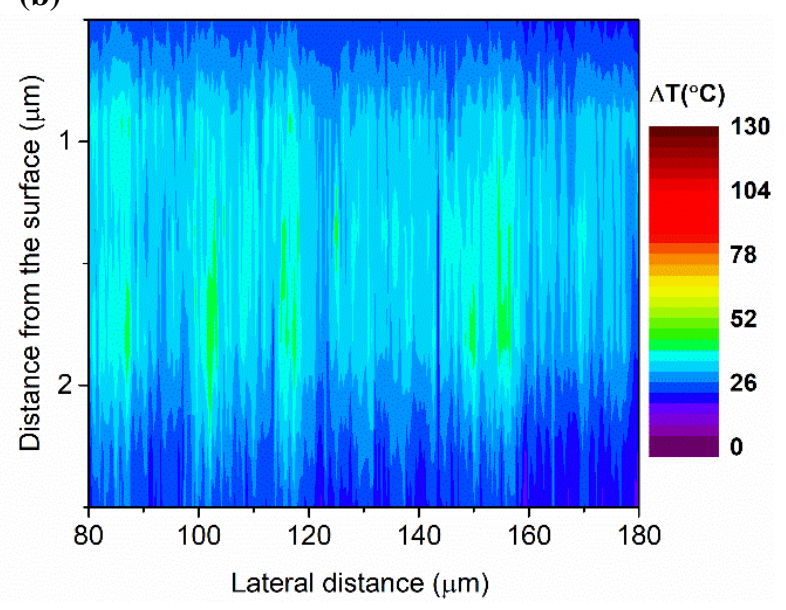

Figure 5. Magnified facet temperature map around the active region for (a) standard laser shows $\Delta \mathrm{T}_{\mathrm{av}}=59 \pm 3.0^{\circ} \mathrm{C}$ with $\Delta \mathrm{T}_{\text {peak }}=130{ }^{\circ} \mathrm{C}$ and (b) two-section laser shows $\Delta \mathrm{T}_{\mathrm{av}}=34 \pm 0.4^{\circ} \mathrm{C}$ with $\Delta \mathrm{T}_{\text {peak }}=45^{\circ} \mathrm{C}$.

Fig. 5 presents the magnified facet temperature map of the active region for both standard and two-section lasers. It shows that the standard laser has large temperature fluctuations $\left(\Delta \mathrm{T}_{\mathrm{av}}=59 \pm 3.0^{\circ} \mathrm{C}\right)$ and two-section laser has more homogenous temperature fluctuations $\left(\Delta \mathrm{T}_{\mathrm{av}}=34 \pm 0.4^{\circ} \mathrm{C}\right)$. Note that the temperature fluctuations in the standard laser reaches $\Delta \mathrm{T}_{\text {peak }}=130$ ${ }^{\circ} \mathrm{C}$ whereas the it is much lower, $\Delta \mathrm{T}_{\text {peak }}=45^{\circ} \mathrm{C}$, in the two-section laser facet. Both the magnitude of the temperature and its fluctuations show that the two-section laser has much cooler and stable facet temperatures than the standard laser.

\section{THERMAL MODELING}

We simulated the temperature distribution of two-section lasers using the heat transfer module of COMSOL. A heat source representing the dissipated power is placed in the active region of $\left(4 \mathrm{~mm}+\mathrm{L}_{\mathrm{win}}\right)$ long laser. The facet absorption is not considered in the simulations as it is difficult to quantify. The thermal resistance of the laser is assumed to be $2.5 \mathrm{~K} / \mathrm{W}$ and $5 \mathrm{~K} / \mathrm{W}$, which represents typical values for broad-area high power laser diodes [2]. Fig. 6(a) shows COMSOL thermal modeling results of the two-section laser with a high heat load of $20 \mathrm{~W}$ that corresponds to a high current operation for such a laser $[2,16]$. For thermal resistance value of $2.5 \mathrm{~K} / \mathrm{W}$, which can be achieved by epi-down soldering, laser section

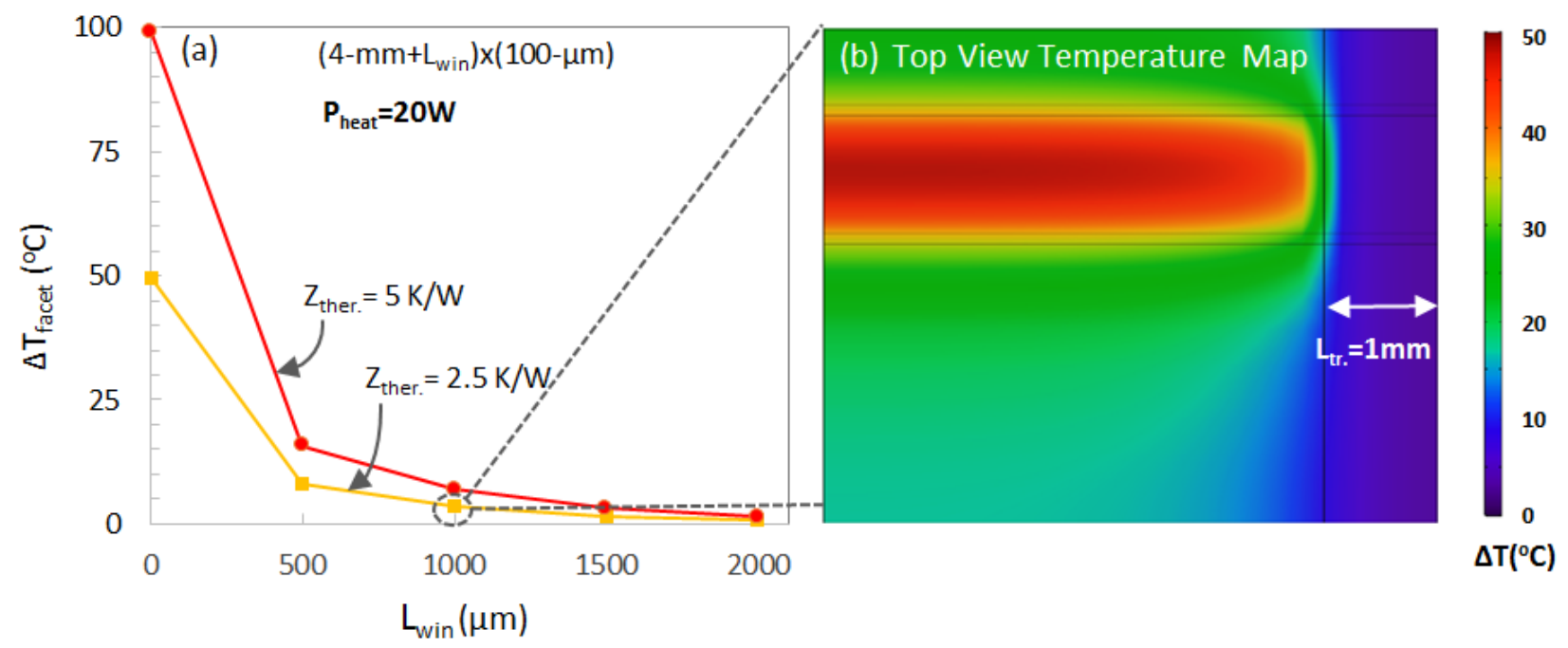

Figure 6. Using a heat load of $20 \mathrm{~W}$, thermal modeling results for (a) facet temperature increase vs. pumped window length for two different thermal resistance values, (b) temperature map for low thermal resistance device with $1 \mathrm{~mm}$ long window. 
contributes less than $1^{\circ} \mathrm{C}$ to the facet temperature using a $1 \mathrm{~mm}$ long transparent window. This is a substantial reduction of the facet temperature compared to $50^{\circ} \mathrm{C}$ for the standard laser diode configuration. It shows that the facet temperature can be reduced by $93-99 \%$ (i.e. up to $\sim 100 \mathrm{x}$ ) by using 1-2 $\mathrm{mm}$ long windows. The top view for the temperature map of this laser is given in Fig. 6(b). As seen from the map, the heat generated by the laser region diffuses longitudinally towards the facet through the cold window. The simulated design is similar to our fabricated lasers and it shows an asymmetric temperature distribution as obtained in the thermoreflectance measurement results presented in Fig. 3(b). As the length of the window section increases, the facet temperature decreases. The data suggest that longer windows are required for higher thermal resistance devices to keep the laser heat contribution to the facet low.

\section{CONCLUSION AND OUTLOOK}

We have demonstrated a promising method for facet temperature reduction for semiconductor lasers using electrically injected transparent windows without compromising the laser performance. The method is based on a monolithic cavity with laser and window sections, where the window is biased to transparency to eliminate its absorption losses. The facet temperature of a two-section laser is reduced even below its laser cavity temperature using a $1 \mathrm{~mm}$ long transparent window. The COMSOL modeling shows that the facet heating due to dissipated power of the lasing region can be almost totally eliminated. The method is general enough to be implemented in a variety of semiconductor lasers and we anticipate that the presented approach could lead to stronger laser facets against COMD and improved device operating lifetime.

\section{REFERENCES}

[1] E. Zucker, D. Zou, L. Zavala, H. Yu, P. Yalamanchili, et al., "Advancements in laser diode chip and packaging technologies for application in kW-class fiber laser pumping," Proc. SPIE 8965, 896507 (2014).

[2] A. Demir, M. Peters, R. Duesterberg, V. Rossin, and E. Zucker, "Semiconductor laser power enhancement by control of gain and power profiles," IEEE Photon. Technol. Lett. 27, 2178 (2015).

[3] V. Gapontsev, et.al, "Highly-Efficient High-Power Pumps for Fiber Lasers," Proc. SPIE 10086, 108604-1 (2017).

[4] J. W. Tomm, M. Ziegler, M. Hempel, and T. Elsaesser, "Mechanisms and fast kinetics of the catastrophic optical damage," Laser Photonics Rev., vol. 5, no. 3, pp. 422-441, 2011.

[5] P. W. Epperlein, "Semiconductor laser engineering, reliability and diagnostics: a practical approach to high power and single mode devices," Wiley, 2013.

[6] P. G. Piva, S. Fafard, M. Dion, M. Buchanan, S. Charbonneau, R. D. Goldberg and I. V. Mitchell, "Reduction of InGaAs/GaAs laser facet temperatures by band gap shifted extended cavities," Appl. Phys. Lett., vol. 70, no. 13, pp. 1662-1664, 1997.

[7] J. Michaud, P. D. Vecchio, L. Béchou, D. Veyrié, M. A. Bettiati, F. Laruelle and S. Grauby, "Precise facet temperature distribution of high-power laser diodes: Unpumped window effect," IEEE Photon. Technol. Lett. 27, 1002 (2015).

[8] F. Rinner, J. Rogg, M. T. Kelemen, M. Mikulla, G. Weimann, J. W. Tomm, E. Thamm and R. Poprawe, "Facet temperature reduction by a current blocking layer at the front facets of high-power InGaAs/AlGaAs lasers," J. Appl. Phys. 93, 1848 (2003).

[9] L. W. Tu, E. F. Schubert, M. Hong and G. J. Zydzik, "In-vacuum cleavingand coating of semiconductor laser facets using thin silicon and a dielectric,” J. Appl. Phys. 80, 6448 (1996).

[10] Patent pending.

[11] S. Arslan, S. Gundogdu, A. Demir, and A. Aydınl, "Facet cooling in high power InGaAs/AlGaAs lasers," IEEE Photon. Tech. Lett. 31, 94 (2019).

[12] S. Arslan, A. Demir, S. Şahin and A. Aydınlı, "Conservation of quantum efficiency in quantum well intermixing by stress engineering with dielectric bilayers," Semicond. Sci. Technol. 33, 025001 (2018).

[13] T. R. Chen, L. E. Eng, Y. H. Zhuang, and A. Yariv, "Experimental determination of transparency current density and estimation of the threshold current of semiconductor quantum well lasers," Appl. Phys. Lett. 56, 1002 (1990).

[14] M. Farzaneh et al., "CCD-based thermoreflectance microscopy: Principles and applications," J. Phys. D: Appl. Phys. 42, 143001 (2009).

[15] D. Pierścińska, "Thermoreflectance spectroscopy-Analysis of thermal processes in semiconductor lasers," J. Phys. D: Appl. Phys. 51, 013001 (2018).

[16] A. Demir, M. Peters, R. Duesterberg, V. Rossin, and E. Zucker, “29.5W continuous wave output from $100 \mu \mathrm{m}$ wide laser diode," Proc. SPIE 9348, 93480G (2015). 